\title{
Preface to the IJAIED 25th Anniversary Issue, Part 2
}

\section{The Next 25 Years: How Advanced Interactive Learning Technologies will Change the World}

\author{
H. Chad Lane ${ }^{1}$ - Gordon McCalla ${ }^{2}$. \\ Chee-Kit Looi ${ }^{3} \cdot$ Susan Bull ${ }^{4}$
}

Published online: 7 March 2016

(C) International Artificial Intelligence in Education Society 2016

\section{AIED: The Next 25 Years}

We are very pleased to present part 2 of the 25th anniversary special issue of the International Journal of Artificial Intelligence in Education (IJAIED) to which we have given the title: The Next 25 Years: How Advanced Interactive Learning Technologies will Change the World. Part 1 of this special issue stands, in the words of the guest editors, as a "celebration of the scholarly kind" of the journal's past 25 years and the $400+$ articles published. It is our honor in part 2 to continue the celebration and share the vision of some of AIED's current and emerging leaders who accepted our invitation to look forward to the next 25 years of learning technology research.

This issue is framed by Part 1 of the special issue, which consists of 35 articles written by authors of the most highly-cited and influential articles from the first 25 years of IJAIED. These authors reflect on their past work, identify its key contributions and impacts, and discuss how the work evolved after the original publication. Importantly, authors were also asked to consider remaining open questions and what would be needed today in order to solve the problems they had originally pursued. It is with these "remaining open questions" that Part 2 of the anniversary issue picks up. We focus on the road ahead for AIED, what new research

H. Chad Lane

hclane@illinois.edu

1 Department of Educational Psychology, University of Illinois at Urbana-Champaign, Champaign, IL, USA

2 Department of Computer Science, University of Saskatchewan, Saskatoon, Saskatchewan, Canada

3 National Institute of Education, Nanyang Technological University, Singapore, Singapore

4 Institute of Education, University College London, London, UK 
directions may emerge, what problems are now within reach, and how AIED research might change the future of learning.

The creation of the journal 25 years ago (and the conference and the society around the

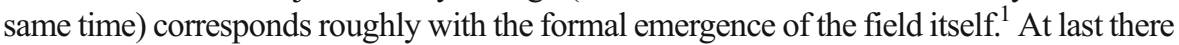
was a community where researchers from many different perspectives could interact to understand the deep and broad issues that had to be resolved to build effective advanced learning technologies. The vision was of a discipline that was fundamentally interdisciplinary, combining advanced technology (the "AI") with advanced social science (the "ED"). A good AIED research project was seen to be one in which both the technological frontier and the pedagogical frontier were being challenged in novel and interesting ways.

We believe that AIED has lived up to this interdisciplinary vision, and this is reflected in the wide diversity of papers in IJAIED. Since its inception 25 years ago, the scope of the journal's research focus has consistently expanded and incorporated ideas from related fields. Although the "AI" in AIED continues to be central to the work of many in the field, new, cross-disciplinary topics of high relevance to education and the learning sciences also continue to find a home in AIED. For example, it is not uncommon to find research described in IJAIED papers that has a strong footing in other areas of study such as affective computing, collaborative learning, educational games, immersive technologies, and more. Many of the systems described in IJAIED articles can still be classified as one-on-one tutors, to be sure, but also there are systems built with different pedagogical assumptions, supporting collaborative learning, communities of learners, exploratory learning, distance education, mobile learning, etc. And it is not just AI technologies that are used, but also work that draws on techniques adapted from data mining, personalization, human-computer interaction, the semantic web, recommender systems, multi-agent systems, information visualization, computational linguistics, simulation, and so on. Methods from social science (particularly cognitive science, education, and anthropology) are commonly used for theoretical insight and for empirical validation, including models of learning, pedagogy, motivation and affect, theory formulation, testing and refinement; and approaches to studying cultural and other social contexts; etc. In short, it is not an accurate characterization to describe AIED as simply AI researchers searching for problems to solve with their "AI hammers", but rather a problem- and research-driven community effort that seeks insights and influence from a wide variety of areas and perspectives.

But, AIED also has some core beliefs. Over the past 25 years three key characteristics of AIED research have emerged that are well worth identifying and building upon: (i) aggressiveness in asking hard and deep questions; (ii) openness to an eclectic range of ideas; and (iii) a learner-first perspective. Indeed, perhaps the most defining "core value" of research in AIED throughout its history is the placement of the learner as the focus of our body of work. AIED research is driven by important problems for which AI in conjunction with a host of other perspectives and disciplines is used to create advanced learning technologies that "care" for the learner (Self 1998).

One of the goals of this special issue is to highlight AIED's interdisciplinary themes and convey their continued importance in the evolution of AIED. We therefore invited authors from different perspectives, including some who are active in other advanced

\footnotetext{
${ }^{1}$ For a more nuanced description of the history of AIED see John Self's article in part 1 of the IJAIED 25th anniversary special issue.
} 
learning technology communities, to write forward looking papers as to the coming key issues and challenges for learning technology. These closely related researchers publish under an alphabet of different labels include the International Society for the Learning Sciences (ISLS); Computer Supported Collaborative Learning (CSCL); Intelligent Tutoring Systems (ITS); Educational Data Mining (EDM); User Modeling, Adaptation, and Personalization (UMAP); the Association for the Advancement of Affective Computing (AAAC); and the International Conference on Computers in Education (ICCE). There are common threads of research connecting these communities. Many researchers are active in more than one of them, and many research projects could easily be categorized as belonging to several of them. We believe that a continued blurring of lines is both desirable and inevitable as we work together to address the daunting and constantly changing challenges of supporting education and learning in the 21 st century.

These are interesting times. When MOOCs burst onto the scene a few years ago, there was an upsurge of interest in learning technology. While MOOC course content could be easily distributed to hundreds or thousands of students, being able to support each of these students, each with their own individual learning needs, was another matter. Suddenly, all of the issues explored by AIED and other advanced learning technology communities over the years were deeply relevant as the focus returned to a student interacting with or through technology to learn. This has led to a renewed optimism about the potential for the widespread impact of learning technology, increased funding from both governments and industry, a coming together of the various advanced learning technology research communities, and the promise of challenging new research questions, interesting new insights, and useful new technologies.

\section{What you will find in this Special Issue}

The 15 papers presented in Part 2 offer some common themes, but also some wide variance on what issues, problems, and challenges we will face as we approach 2041. One conspicuous area of difference lies in the authors' comfort in writing about the future. While some authors were wary of the idea of making long term predictions, others have chosen to boldly do so. Although we did not specifically request predictions, we did ask authors for their opinions on what may happen in AIED research and "to imagine what directions our field may take and to frame the research we will do over the next quarter century." Alan Kay, computer science pioneer and one of the earliest educational technology researchers, famously said "The best way to predict the future is to invent it." (Gasch and Kay, 1996). In this special issue, we have embraced this challenge. We hope that the papers will not only impact AIED and related learning technology communities, but will also signal to the rest of the technology and education worlds what we hope to do to improve learning and education going forward.

In deciding how to organize the special issue, we opted to wait for near-final versions of the articles to see if obvious groupings emerged naturally. Indeed, we were pleasantly surprised to see that this did in fact happen. Specifically, we found four categories of papers that are organized in roughly chronological terms. These focus on (1) what AIED history has to say about its future, (2) existing research problems and thrusts that are poised to continue to be important, (3) new directions and problems that authors believe AIED researchers should pursue, and (4) what potential futures lie 
ahead and how rapid changes in the world may influence the research we choose to pursue.

This special issue begins with a set of papers that form a bridge between our past and future by focusing on the roots and underpinnings of AIED research and showing how the past will be prologue to the future. Dillenbourg's paper explores the historical roots of AIED and identifies six trends that highlight the increasingly interdisciplinary nature of AIED. He suggests these trends will only gain in importance as the world presents problems of concomitant complexity. Kinshuk, Chen, Cheng, and Chew also provide a historical framing, but focus directly on a vision of building smarter learning environments and their potential roles in an expanding ecosystem of intelligent learning technologies. Next, in a very reflective piece, Roll and Wylie report on their review of 47 IJAIED papers taken from 1994, 2004, and 2014. They conclude by recommending two broad strands of research, one that is evolutionary (in that we should work more closely with existing educational practices) and one that is revolutionary and disruptive (to embed AIED technologies in brand new contexts that are quickly gaining relevance with today's learners).

Our second set of papers fall into the "everything that is old is new again" category by covering topics in AIED that have received substantial attention, but are argued to deserve continued and renewed focus moving forward. Baker presents the argument for a data-driven approach towards personalized learning systems and outlines new directions for educational data mining research with an emphasis on modeling and feedback. Heffernan, et al. present a different, but very much complementary vision for datadriven systems through "learnersourcing" and "teachersourcing". In D'Mello's vision of the next 25 years, AIED systems will extend modeling and monitoring of various aspects of the learner and the learning experience to include the learners' attentional state. The paper proposes research on attention tracking and management that is directed towards dynamically directing attention for highly effective learning. Rose and Ferschke round out this second set of papers by looking at a venerable, but powerful pedagogical tool: discussion. Focused on the next decade of research, they highlight the deep links between natural language processing, AIED, and collaborative learning. Their vision goes beyond single learning episodes and proposes shifts to learning communities and communities of practice.

The next 25 years will most likely bring about many new challenges and surprises, which implies AIED research will also need to evolve to address important new competencies and learning contexts. The third set of papers includes papers that make this assumption by suggesting new research directions that AIED should pursue. Porayska-Pomsta argues that a critical direction for AIED research is "educational praxis", determining how to support the lifelong learning needs of teachers. She looks forward to a large role for AI in providing teachers with appropriate scaffolding for enhancing their meta-cognitive skills in support of their teaching and their students' learning. Timms envisions an increase in the role of tangible systems in education, and argues that these new systems (some of which he calls "educational co-bots") will need enriched models of their surroundings, pedagogical roles, and purpose. His argument is that $\mathrm{AI}$ advances are just as important as advances in robotics. Complementing Timm's vision, Walker and Ogan draw on compelling evidence from the social sciences by arguing that a social perspective on learning must underlie the next 25 years of research. Specifically, they propose that AIED systems should be 
built with the relationship with the learner playing a central role. They effectively define a modernized view of Self's transformative call for "systems that care" (Self 1998). Santos rounds out the section by calling for an expansion of AIED research into motor skill learning. She argues that part of the value in this endeavor will be to revisit AIED's roots and to highlight the powerful potential marriage between AIED and existing (non-adaptive) systems for motor skill learning.

The special issue culminates with four articles that present bold and specific possibilities for the future of AIED and educational technologies. They point in new directions that, if pursued, may radically alter the positioning of AIED research in the future of learning. Nye begins this final section by proposing a vision of an ecosystem of interacting learning tools that span different and diverse levels of adaptation ranging from selecting different learning systems to adaptation at the step or micro level of learning. This framing can support new experimentation in education, reframing research questions to study design principles based on this overall view of a serviceoriented architecture and adaptive systems. Pinkwart similarly embraces the call to envision the next 25 years by presenting both utopian and dystopian visions for the future, leading to seven grand challenges for AIED. Rummel, Walker, and Aleven also adopt a dystopian/utopian approach, but focus squarely on the roles of technology in collaborative and individual learning. They argue that there is an increasing need for a deeper consideration of theoretical models of collaboration, both in the application and in the design of adaptive supports for learning. Burleson and Lewis bring the special issue to a close by writing from the perspective of year 2041. Their intriguing vision encapsulates a 50-year reflection on AIED by imagining a true science fiction-eligible future with deeply connected learning resources, full sensory immersion technologies, true personalization, and scale beyond any conception available to us today.

While we have divided these papers into four sections because we have to put them in some order, the papers do, of course, transcend such simple categorization. Common themes weave among them, all authors look both to the past and the future, new ideas flow from them all. As guest editors, we hope you will enjoy reading the papers in Part 2 of the special issue celebrating the 25th anniversary of the International Journal of Artificial Intelligence in Education. Hopefully, between the two parts, you will be reminded of the substantial accomplishments of the AIED field over the past 25 years, will celebrate the deep and diverse interconnectivity of the ideas underlying AIED, and will be stimulated in new and interesting directions as AIED moves forward.

\section{References}

Gasch, S., and Kay, A. (1996). Alan Kay. Interactive learning with a digital library in computer science project. http://s3.amazonaws.com/lcp/universidad-intercontinental/myfiles/Alan\%20Kay.pdf. Accessed Jan 312016.

Self, J. (1998). The defining characteristics of intelligent tutoring systems research: ITSs care, precisely. International Journal of Artificial Intelligence in Education (IJAIED), 10, 350-364. 